\title{
Characterization of immunogenic proteins of Cysticercus tenuicollis of goats
}

\author{
A. Goswami, M. Das and R. Laha \\ Division of Veterinary Parasitology \\ ICAR Research Complex for NEH Region \\ Umroi Road, Umiam, Meghalaya 793 103, India \\ Corresponding author: R. Laha, email:rglaha@gmail.com \\ Received: 11-08-2012, Accepted: 01-10-2012, Published online: 07-02-2013
}

\section{How to cite this article:}

Goswami A, Das M and Laha R (2013) Characterization of immunogenic proteins of Cysticercus tenuicollis of goats, Vet. World 6(5): 267-270, doi: 10.5455/vetworld.2013.267-270

\begin{abstract}
Aim: To identify immunodominant proteins of cystic fluid antigens and whole cyst lysate antigens of Cysticercus tenuicollis, the larval stage of the canine tapeworm Taenia hydatigena.

Materials and Methods: Three numbers of cysts of $C$. tenuicollis were collected from the mesentry of small intestine of goats after slaughter. C. tenuicollis cysts of each sample were washed thoroughly with PBS (pH 7.4). Two types of antigens i.e. cystic fluid antigens and whole cyst lysate antigens were prepared from each sample. Polypeptide profiles of cystic fluid antigens and whole cyst lysate antigens preparations of $C$. tunicollis were analysed by Sodium dodecyl sulphatepolyacrylamide gel electrophoresis (SDS-PAGE) containing 10\% gel. C. tenuicollis proteins resolved by SDS-PAGE were electrophoretically transferred from the gel to a nitrocellulose membrane and probed by western blotting using natural immune sera obtained from infected goats. C. tenuicollis proteins that were resolved by SDS-PAGE and reactive by immune sera of goat was estimated by a Soft ware programme named KODAK 1D Image Analysis Software.

Results: A total of eight major polypeptides of molecular weight $(\mathrm{Mr}) 149.4 \mathrm{kDa}, 92.9 \mathrm{kDa}, 74.2 \mathrm{kDa}, 63.5 \mathrm{kDa}, 36.2 \mathrm{kDa}$, $23.9 \mathrm{kDa}, 15.7 \mathrm{kDa}$ and $9.6 \mathrm{kDa}$ were resolved by SDS-PAGE with minor variations. Out of these, three polypeptides of $\mathrm{Mr}$ $36.2 \mathrm{kDa}, 23.9 \mathrm{kDa}$ and $9.6 \mathrm{kDa}$ were recognized as immunodominant polypeptides after western blotting. Both cystic fluid antigens and whole cyst lysate antigens resolved same $\mathrm{Mr}$ polypeptides by SDS-PAGE and identified same immunodominant polypeptides after western blotting. The immunodominant polipeptides of $M r 23.9 \mathrm{kDa}$ and $9.6 \mathrm{kDa}$ identified for the first time from both cystic fluid antigens and whole cyst lysate antigens prepared from Cysticercus tenuicollis.
\end{abstract}

Conclusion: Eight polypeptides were resolved from cystic fluid antigens and whole cyst lysate antigens of Cysticercus tenuicollis by SDS-PAGe of which polypeptides of three polypeptides of $M r 36.2 \mathrm{kDa}, 23.9 \mathrm{kDa}$ and $9.6 \mathrm{kDa}$ were recognized as immunodominant polypeptides after western blotting. These three immunodominant polypeptides could be explored for sero diagnosis of $C$. tenuicollis infection in goats.

Keywords: Cysticercus tenuicollis, goat, immunogenic, polypeptide

\section{I ntroduction}

The larval stage of the canine tapeworm Taenia hydatigena inside their intermediate host is known as Cysticercus tenuicollis. The infection has been reported to present in wild and domestic ruminants throughout the world. The morphological features are used to identify the cyst but now-a-days molecular tools are being used to define species of parasites in cases where the key morphologic features can not detect species of parasites [1]. The infection causes condemnation of meat and thereby economic losses [2, $3]$. It is generally found in the mesentries, omenta, cavities and also in the liver, lungs, kidneys and brain of infected animals [4,5]. Aberrant location of $C$. tenuicollis inside the chorion-allantoic membrane of goats has also been reported [6]. C. tenuicollis infection in liver causes hepatitis, burrowing canal, granular degeneration, deposition of serofibrinous exudates and in lungs it causes pneumonitis $[4,7]$. Besides this pathogenicity, outbreaks due to acute Cysticercus tenuicollis in goats have been reported from goats of India and abroad $[4,8]$. High prevalence of infection $(46.6 \%, 55.77 \%$ and $63.9 \%)$ have been reported in goats from Ethiopia and Benin, after abattoir study and adult goats were found more infected than kids $[3,5,9]$. Cysticercus tenuicollis cysts were found in $18.04 \%$ goats of Iran [10] and $10 \%$ goats of Iraq [11].

Almost similar percentage of infection (18.75\%) in goats has been reported from Maharashtra State, India [12]. The prevalence of $C$. tenuicollis infection in goats from Durg, Chattishgarh was reported as $21.01 \%$ [13]. There is no effect of season on prevalence of this infection [5]. Significant level of calcium, sodium, potassium and considerable amount of Asparate aminotransferase (AST), Alkaline phosphatase (ALKP), Lactate dehydrogenase (LDH) with low concentration of Alanine aminotransferase have been reported to be present in cystic fluid of C. tenuicollis of goats [14]. Examination of post mortem materials is the usual method of diagnosis. Detection of antibodies could be of interest as a tool for immunodiagnosis of the 


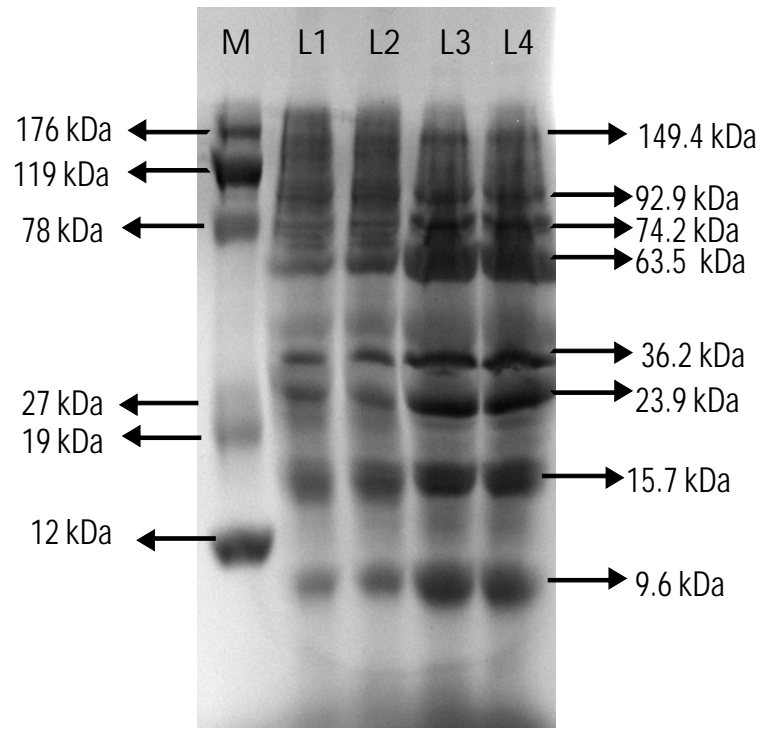

Figure-1. Polypeptide profiles of cystic fluid antigens (L1\&L2) and whole cyst lysate antigens (L3\&L4) prepared from $C$. tenuicollis, resolved in 10\% SDS-PAGE and stained with CBB. Lane M-Standard Molecular Weight Marker

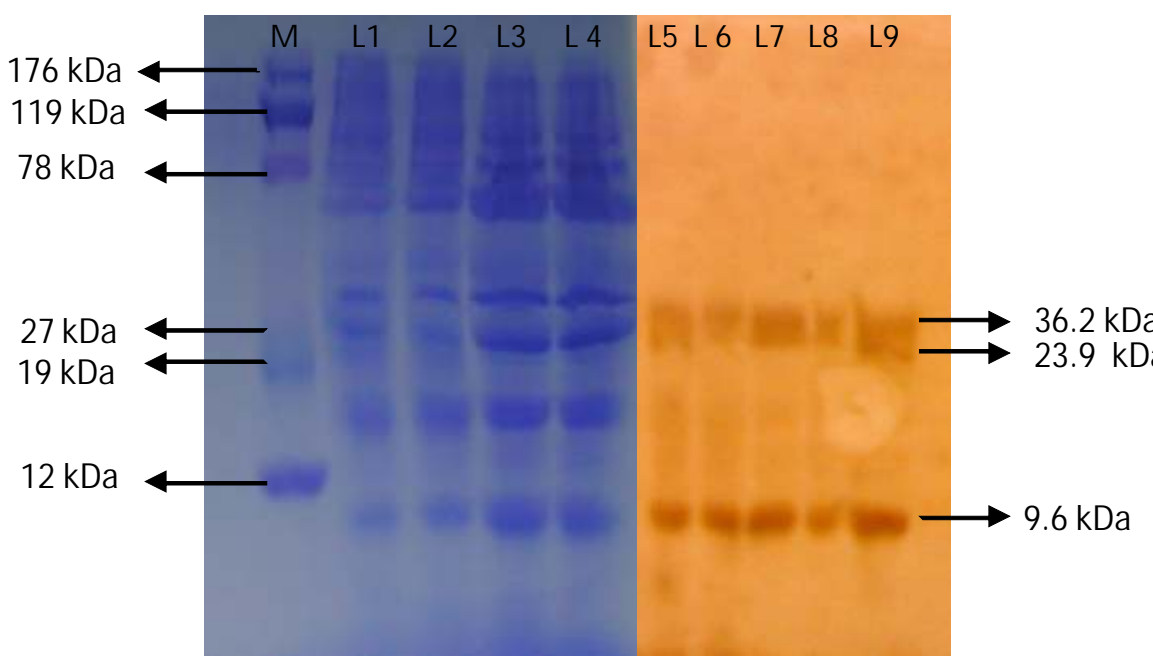

Figure-2. Polypeptide profiles of cystic fluid antigens (L1\&L2) and whole cyst lysate antigens (L3\&L4) prepared from C.tenuicollis, resolved in $10 \%$ SDS-PAGE and stained with CBB (Left Portion). Western blot analysis of the same. Lane L5 to L7 whole cyst lysate antigens; L8 \& L9 cystic fluid antigens (Right Portion). Lane M-Standard Molecular Weight Marker

infection. For this it is essential to know about the immunogenic proteins of $C$. tenuicollis. Hence, here an attempt has been taken to identify the immunogenic proteins of $C$. tenuicollis using SDS-PAGE analysis followed by western blotting of both cystic fluid antigens and whole cyst antigens, as such type of reports are very scanty from India $[15,16]$ and also reports are not available from North Eastern region of India.

The present study was aimed to identify immunodominant proteins of cystic fluid antigens and whole cyst lysate antigens of Cysticercus tenuicollis, the larval stage of the canine tapeworm Taenia hydatigena.

\section{Materials and Methods}

Collection of cyst and preparation of antigen: Three numbers of cysts of $C$. tenuicollis were collected from the mesentry of small intestine of goats after slaughter. No other helminth parasitic infection was noticed after post mortem examination of these animals. $C$. tenuicollis cysts of each sample were washed thoroughly with PBS ( $\mathrm{pH}$ 7.4). Two types of antigens i.e. cystic fluid antigens and whole cyst lysate antigens were prepared from each sample. For preparation of cystic fluid antigens, the fluid of each cyst was aspirated from the cyst with the help of a sterilized syringe and needle and collected directly in centrifuge tube. It was centrifuged at $15000 \mathrm{xg}$ for 15 minutes at $4^{\circ} \mathrm{C}$. Supernatant was concentrated for two times using Nanosep centrifugal device, 10k Omega membrane, blue (Cat. No. CLNT10C34, Clonitec) and treated as cystic fluid antigen. After removing the vesicular fluid, whole cyst lysate antigen was prepared. The cyst membrane along with the scolex was homogenized in a sterilized pestle and mortar after adding $3 \mathrm{ml}$ of PBS ( $\mathrm{pH}$ 7.4). The homogenate was further subjected to sonication (B. Braun Biotech International, Sartoerious group) for three times for a period of 30 seconds with 30 seconds cooling interval each time at $30 \mathrm{kHz}$ on an ice bath. After sonication, the homogenate was centrifuged at $15000 \mathrm{xg}$ for 15 minutes at $4^{\circ} \mathrm{C}$. The supernatant fluid was concentrated for two times using Nanosep centrifugal device, 10k Omega membrane, blue (Cat. No. CLNT10C34, Clonitec) which was treated as whole cyst antigen. The protein content of these supernatant fraction was measured as per standard method [17] using BSA as the standard. The concentration of protein in the supernatant of fluid Ags 
and whole cyst Ags were estimated as $2.8 \mathrm{mg} / \mathrm{ml}$ and $3.2 \mathrm{mg} / \mathrm{ml}$ respectively. The antigens, thus prepared were stored in aliquots at $-20^{\circ} \mathrm{C}$ till further use.

Source of immune sera: During slaughter, blood samples of the goats were collected in sterile condition and sera of the goats which were found positive for $C$. tenuicollis infection were preserved at $-20^{\circ} \mathrm{C}$ for further use at the time of immunoblot.

SDS-PAGE and western blot analysis: For identification of immunogenic proteins, polypeptide profiles of cystic fluid antigens and whole cyst lysate antigen preparations of each $C$. tenuicollis were analysed by SDS-PAGE (Genei Bangalore, India) containing 10\% gel under protein denaturing condition [18]. Standard molecular weight marker protein (Lonza, USA) was run simultaneously. Two separate gels for each sample were run in same conditions using the same antigen samples prepared from C. tenuicollis. After completion of running of the SDS-PAGE, one gel along with molecular weight marker was shifted to stain with Coomassie Brilliant Blue. Remaining one was washed with transfer buffer and $C$. tenuicollis proteins resolved by SDS-PAGE were electrophoretically transferred (Genei, Bangalore) from the gel to a nitrocellulose membrane as per the standard method [19] and probed by western blotting using natural immune sera obtained from infected goats. The $M r$ for the $C$. tenuicollis proteins that were resolved by SDS-PAGE and reactive by immune sera of goat was estimated by a Soft ware programme named KODAK 1D Image Analysis Software.

\section{Results}

The polypeptide profiles of cystic fluid antigens and whole cyst lysate antigens as resolved by SDSPAGE have been shown in Fig-1. A total of eight polypeptides of $M r 149.4 \mathrm{kDa}, 92.9 \mathrm{kDa}, 74.2 \mathrm{kDa}$, $63.5 \mathrm{kDa}, 36.2 \mathrm{kDa}, 23.9 \mathrm{kDa}, 15.7 \mathrm{kDa}$ and $9.6 \mathrm{kDa}$ were resolved by SDS-PAGE from each samples with minor variations. Out of these three polypeptides of $\mathrm{Mr}$ $36.2 \mathrm{kDa}, 23.9 \mathrm{kDa}$ and $9.6 \mathrm{kDa}$ were recognized as immunodominant polypeptides after western blotting. All of them could be considered as major polypeptides. It could also be observed that both cystic fluid antigens and whole cyst lysate antigens resolved same $\mathrm{Mr}$ polypeptides. Fig- 2 represent the Western blot analysis for identification of corresponding immuno-genic proteins using immune sera. A total of three polypeptides of $M r 36.2 \mathrm{kDa}, 23.9 \mathrm{kDa}$ and $9.6 \mathrm{kDa}$ were recognized as immunodominant polypeptides from each samples with minor variations. This was also found same for both cystic fluid antigens and whole cyst lysate antigens.

\section{Discussion}

Analysis of polypeptides in 10\% SDS-PAGE of both cystic fluid antigens and whole cyst lysate antigens revealed a complex protein band pattern with the appearance of almost similar molecular weight polypeptides for both Ags, might be due to their same composition. The slight difference may be due to the host variation. Although eight polypeptides were resolved after SDS-PAGE analysis, only three of them have been found immunogenic in the present study, for both the Ags. This indicated that immunogenecity of both Ags are same. Thus three immunodominant polypeptides of $M r 36.2 \mathrm{kDa}, 23.9 \mathrm{kDa}$ and $9.6 \mathrm{kDa}$ with minor variation were identified from both cystic fluid antigens and whole cyst lysate antigens. In a study, only one immuno reactive protein band of $\mathrm{Mr} 36$ $\mathrm{kDa}$ has been reported from Turkey [20] and almost similar immunodominant polypeptide of $\mathrm{Mr} 36.2 \mathrm{kDa}$ also identified in the present study. The additional twoimmunodominant polypeptides of $M r 23.9 \mathrm{kDa}$ and 9.6 $\mathrm{kDa}$ as identified in the present study might be due to antigenic variation of the metacestode due to their strain variation and geographical distribution. These immunogenic antigens could be used for immunodiagnosis of C. tenuicollis after purification using the technique either counter immunoelectrophoresis (CIEP) or agar gel precipitation test (AGPT) in the field. The protein profiles of dialyzed cyst fluid, cyst membrane and scolex of $C$. tenuicollis resolved a total 37 bands by SDS-PAGE, out of which 3 bands were observed in the cyst fluid, 22 in the cyst membrane and 12 bands in the scolex of the cyst [15]. They observed cyst fluid proteins were least reactive, whereas membrane proteins elicited a consistent reaction with the hyperimmune sera after western blot analysis. In a recent study from India, cystic membrane antigens of C. tenuicollis revealed 15 polypeptide bands of $\mathrm{Mr} 10$ to $131 \mathrm{kDa}$ by SDS-PAGE analysis where naturally infected goat serum identified two immunodominant bands of $\mathrm{Mr} 34 \mathrm{kDa}$ and $14 \mathrm{kDa}$ [16] and they concluded that these two bands could be explored for sero diagnosis of $T$. hydatigena cysticercosis in goats.

\section{Conclusion}

Eight polypeptides were resolved from cystic fluid antigens and whole cyst lysate antigens of Cysticercus tenuicollis by SDS-PA.Ge of which polypeptides of three polypeptides of $M r 36.2 \mathrm{kDa}$, $23.9 \mathrm{kDa}$ and $9.6 \mathrm{kDa}$ were recognized as immunodominant polypeptides after western blotting. These three immunodominant polypeptides could be explored for sero diagnosis of $C$. tenuicollis infection in goats.

\section{Authors' contribution}

All authors contributed equally. All authors read and approved the final manuscript.

\section{Acknowledgements}

Authors are thankful to the Director, ICAR Research Complex for NEH Region, Umroi Road, Umiam, Meghalaya - 793103 , for providing the facilities to carry out this investigation. 


\section{Competing interests}

Authors declare that they have no competing interests.

\section{References}

1. Utuk, A.E., Piskin, F.C. (2012) Molecular Detection and Characterization of Goat Isolate of Taenia hydatigena in Turkey. The Scientific World J. 2012: 1-4.

2. Jibat, T., Ejeta, G., Asfaw, Y., Wudie, A. (2008) Causes of abattoir condemnation in apparently healthy slaughtered sheep and goats at HELMEX abattoir, Debre Zeit, Ethiopia. Revue Med. Vet., 159: 305-311.

3. Wondimu, A., Abera, D., Hailu, Y. (2011) A study on the prevalence, distributionand economic importance of Cysticercus tenuicollis in visceral organs of small ruminants slaughtered at an abattoir in Ethiopia. J. Vet. Med. Anim. Health, 3: 67-74.

4. Shivasharanappa, N., Gururaj, K., Sharma, D.K., Reddy, G.B.,Manjunatha, V. (2011) Acute hepatic and pneumonic cysticercosis in Barbari goats. Ind. J. Vet. Path., 35: 80-81.

5. Attindehou, S.,Salifou, S. (2012) Epidemiology of Cestodes Infections in Sheep and Goats in Benin. Vet. Res., 5: 59-62.

6. Payan-Carreira, R., Silva, F., Rodrigues, M.,dos Anjos Pires, M.(2008) Cysticercus tenuicollis vesicle in fetal structures: report of a case. Reproduction in Domestic Animals, 43:764766.

7. Nath, S., Pal, S., Sanyal, P.K., Ghosh, R.C., Mandal, S.C. (2011) Histopathology of hepatitis cysticercosa in goat. $J$. Vet. Parasitol, 25:187-188.

8. Manfredi, M.T., Ghirardelli, R., Zanzani, S. (2006) Cysticercus tenuicollis infection in a goat farm. Parasitologia, 48: 433-436.

9. Samuel, W., Zewde, G.G. (2010). Prevalence, risk factors, and distribution of Cysticercus tenuicollis in visceral organs of slaughtered sheep and goats in central Ethiopia. Trop. Anim. Health Prod.42: 1049-1051.

10. Radfar, M. H., Tajalli, S., Jalalzadeh, M. (2005) Prevalence and morphological characterization of Cysticercus tenuicollis (Taenia hydatigena cysticerci) from sheep and goats in Iran. Vet. Arhiv. 75: 469-476.

11. Al-Bakri, H.S. (2012) Prevalence of Tenuicollosis Among Livestock Slaughtered at Ninevah Governorate-Iraq. 3a. J. Adv. Biomed. Pathobiol. Res. 2:30-39.

12. Nimbalkar, R.K., Shinde, S.S., Kamtikar, V.N., Muley, S.P. (2011) Study on Taenia hydatigena in Slaughtered Sheep (Ovis bharal) and Goats (Capra hircus) in Maharastra, India. Global Veterinaria, 6: 374-377.

13. Nath, S., Pal, S., Sanyal, P.K., Mandal, S.C. (2009) Prevalence of caprine Taenia hydatigena cysticercosis in Durg, Chattisgarh. Ind. J. Field Vet., 5:64-66.

14. Nath, S., Pal, S., Sanyal, P.K., Ghosh, R.C., Mandal, S.C. (2010) Chemical and Biochemical characterization of Taenia hydatigena cysticerci in goats Vet. World. 3: 312-314.

15. Mathur, V.C., Aiyasami, S.S., Latha, B.R., John, L. (2003) Western blot analyses of Cysticercus tenuicollis antigens. Ind. J. Anim. Sci. 73: 837-839.

16. Nath, S., Pal, S., Sanyal, P.K., Mandal, S.C. (2011) Determination of immune reactive proteins in cystic membrane of Cysticercus tenuicollis of goat origin. Ind. J. Anim. Sci., 81: 822-823.

17. Lowry, O.H., Rosebrough, N.J., Farr, A.B., Randall, R.J. (1951) Protein measurement with the Folin-phenol reagent. Journal of Biological Chemistry, $193: 265$.

18. Laemmli, U.K. (1970) Cleavage of structural proteins during the assembly of the head of bacteriophage T4. Nature, $227: 680-685$.

19. Towbin, H., Staehelin, T., Gordon, J. (1979) Electrophoresis transfer of proteins from polyacrylamide gels to nitrocellulose sheets : procedure and some applications. Proceedings of the National Academy of Sciences, USA, 76 : 4350-4345.

20. Kara, M., Sarimehmetoglu, H.O., Gonenc, B. (2003) The determination of immune reactive proteins in Cysticercus tenuicollis cyst fluids by SDS-PAGE and western blotting in sheep. Parasite, 10 :141-145. 